\title{
Der Landesfürst als Lehensherr. Urkundliche Quellen zum Lehenswesen in den habsburgischen Erblanden des Spätmittelalters
}

\section{The landlord as a beneficial owner. Charters documenting fiefdom in the hereditary territories of the Habsburgs during the late middle ages}

Daniel Luger / daniel.luger@univie.ac.at

Institut für österreichische Geschichtsforschung, Universität Wien

\begin{abstract}
The study is dedicated to an reappraisal of the sovereign's fief policy in late middle age Austria. While the older research tendencies were mostly based on the idea of a slightly declining system signed by a rigid formalism, a new analysis of the fief structures in the hereditary territories of the Habsburg dynasty shows a slightly different picture. The anatomy of fief letters and books, reverses and letters of conveyance show that the increasing literacy transformed fiefdom into one of the central playgrounds of sovereign chancelleries. Mostly in case of dominion divisions or changes in late medieval rulership that vassality became important. Despite the reification of fiefdom, territorial rule and vasality do not oppose each other. The sovereign fiefdom in the 14 th and 15 th century is considered as an important often monetarised instrument among others, used to enhance and consolidate the sovereign's rule.
\end{abstract}

\section{Keywords}

Fiefdom, Territorialisation, Austria, Habsburg, Late Middle ages 
Während für einige größere und kleinere Fürstentümer des römisch-deutschen Reiches umfassende Studien zu landesfürstlicher Lehenspolitik und -verwaltung im Spätmittelalter sowie zum territorialen Lehensrecht vorhanden sind, ${ }^{1}$ ist man hinsichtlich der habsburgischen Erblande bislang nicht über einige wertvolle, räumlich und quellenmäßig allerdings deutlich eingeschränkte Einzelstudien hinausgekommen. ${ }^{2}$

Das weitgehende Desinteresse der österreichischen Forschung wird nicht zuletzt in Otto Brunners bis heute einflussreichem Werk „Land und Herrschaft“ dokumentiert, in dem der Autor dem landesfürstlichen Lehenswesen lediglich sechs der über 450 Seiten seiner Studie widmet, denn dieses besitze nach Brunner in den mittelalterlichen österreichischen Ländern „nicht jene Bedeutung, die man ihm, offenbar durch die Bedeutung des Lehnrechtes für die Reichsverfassung und die geringe Erforschung der territorialen lehenrechtlichen Verhältnisse verleitet, häufig zuschreibt". ${ }^{3}$ Die vermeintliche Irrelevanz des territorialen Lehenswesens in Österreich begründet Brunner mit

1 Vgl. etwa die grundlegenden Arbeiten von Diestelkamp, Bernhard: Das Lehnrecht der Grafschaft Katzenelnbogen, 13. Jahrhundert bis 1479. Ein Beitrag zur Geschichte des spätmittelalterlichen deutschen Lehnrechts, insbesondere zu seiner Auseinandersetzung mit oberitalienischen Rechtsvorstellungen. Untersuchungen zur deutschen Staats- und Rechtsgeschichte N.F. 11. Aalen 1969; ders.: Lehnrecht und spätmittelalterliche Territorien. In: Der deutsche Territorialstaat im 14. Jahrhundert. Band 1. Hg. von H. Patze. Vorträge und Forschungen 13, 1970, S. 65-96; ders.: Lehnrecht und Lehnspolitik als Mittel des Territorialausbaus. Rheinische Vierteljahresblätter 63, 1999, S. 26-38; sowie Spieß, Karl-Heinz: Lehnsrecht, Lehnspolitik und Lehnsverwaltung der Pfalzgrafen bei Rhein im Spätmittelalter. Geschichtliche Landeskunde 18. Wiesbaden 1978; ders.: Das Lehnswesen in Deutschland im hohen und späten Mittelalter. Stuttgart 2011², bes. S. 49-62. Zu anderen Territorien des Reiches siehe etwa Weizsäcker, Wilhelm: Über die Bedeutung des Lehnswesens in den Sudetenländern. In: Studien zum mittelalterlichen Lehenswesen. Vorträge gehalten in Lindau am 10.-13. Oktober 1956. Vorträge und Forschungen 5. Lindau - Konstanz 1960, S. 229-234; Theuerkauf, Gerhard: Land und Lehnswesen vom 14. bis zum 16. Jahrhundert. Ein Beitrag zur Verfassung des Hochstifts Münster und zum nordwestdeutschen Lehnrecht. Neue Münstersche Beiträge zur Geschichtsforschung 7. Köln - Graz 1961; Miller, Matthias: Mit Brief und Revers. Das Lehenswesen Württembergs im Spätmittelalter. Quellen - Funktion - Topographie. Schriften zur südwestdeutschen Landeskunde 52. Leinfelden-Echterdingen 2004. Zu den Wurzeln des territorialen Lehenswesens im Hochmittelalter siehe Andermann, Kurt: Verbreitung, Strukturen und Funktion des Lehnswesens im Umkreis von Fürsten, Grafen, Herren und Prälaten vom 11. bis in die Mitte des 13. Jahrhunderts. In: Ausbildung und Verbreitung des Lehnswesens im Reich und in Italien im 12. und 13. Jahrhundert. Hg. von K.-H. Spieß. Vorträge und Forschungen 76. Ostfildern 2013, S. 307-336.

2 Siehe etwa Karl Lechners eingehende Studien zu den Wegen und Zielen der landesfürstlichen Lehenspolitik im Waldviertel: ders.: Siedlungs- und Herrschaftsgeschichte des Waldviertels. In: Das Waldviertel. Hg. von E. Stepan. Band 7/2. Wien 1937, S. 5-276; ders.: Die Bildung des Territoriums und die Durchsetzung der Territorialhoheit im Raum des östlichen Österreich. In: Der deutsche Territorialstaat im 14. Jahrhundert. Bd. 2. Hg. von H. Patze. Vorträge und Forschungen 14, 1971, S. 389-462, hier: 427f. Vgl. auch Prausnitz, Otto: Feuda extra curtem, mit besonderer Berücksichtigung der Brandenburgischen Lehen in Österreich. Quellen und Studien zur Verfassungsgeschichte des deutschen Reiches in Mittelalter und Neuzeit 6/3. Weimar 1929, sowie Starzer, Albrecht: Die landesfürstlichen Lehen in der Steiermark von 1421-1546. Beiträge zur Kunde steiermärkischer Geschichtsquellen 32, 1903. Zur Lehenspolitik Herzog Rudolfs IV. als Herrschaftsinstrument und Mittel zur Integration seiner Länder und Territorien siehe Hageneder, Othmar: Das Land der Abtei und die Grafschaft Schaunberg. Mitteilungen des Oberösterreichischen Landesarchivs 7, 1960, S. 252-295, bes. 277-280; Lackner, Christian: Das Haus Österreich und seine Länder im Spätmittelalter. Dynastische Integration und regionale Identitäten. In: Fragen der politischen Integration im mittelalterlichen Europa. Hg. von W. Maleczek. Vorträge und Forschungen 63. Ostfildern 2005, S. 278f. (mit weiterführenden Literaturangaben).

3 Brunner, Otto: Land und Herrschaft. Grundfragen der territorialen Verfassungsgeschichte Österreichs im Mittelalter. Wien $1965^{5}$, S. 354-356 und 370-372, hier 355. 
einem argumentum ex silentio: „Schon allein die Tatsache, daß die so außerordentlich eindringende landeskundliche Forschung sich kaum um das Lehnswesen bekümmert hat, weist darauf hin, daß es für das innere Gefüge des Territoriums nicht von entscheidendem Belang ist“. A Allerdings räumt Brunner ein, dass der Landesfürst seit dem späten 13. Jahrhundert gezielt die Erweiterung seiner Lehenshoheit gesucht habe, um durch aufgetragenes Eigengut landsässiger Herren und Ritter sowie das Ausschalten reichsunmittelbarer Lehensherren eine Vermehrung des landesfürstlichen Kammergutes und Blutbannbezirks zu erreichen. ${ }^{5}$

Ausgehend von einem Überblick über die relevante, vor allem urkundliche Quellenüberlieferung soll im Rahmen des folgenden Beitrages eine Reihe bislang wenig beachteter Aspekte des landesfürstlichen Lehenswesen in den habsburgischen Erblanden erörtert und damit ein Beitrag zu einer Neubewertung der spätmittelalterlichen Lehenspolitik der Habsburger in Österreich geleistet werden. ${ }^{6}$

Das europäische Lehenswesen des Spätmittelalters weist bekanntermaßen einige grundlegende strukturelle Neuerungen auf, wobei hinsichtlich der hier behandelten Fragestellung insbesondere das Eindringen der Schriftlichkeit in diesen ursprünglich schrift- und urkundenfreien Bereich von zentraler Bedeutung ist. ${ }^{7}$ Diese europaweit festzustellende Entwicklung kann als Reaktion auf zunehmend komplexer werdende

4 Ebd.

5 Ebd., S. 371f. Nach Ernst Klebel habe das Lehenswesen in Österreich im 14. und frühen 15. Jahrhundert eine gewisse Bedeutung für die Verfestigung des Territorialstaates gehabt, sei allerdings bald danach zu einer Formalität herabgesunken, „die trotz ihrer Bedeutungslosigkeit zäh von seiten der Staatsoberhäupter festgehalten wurde“: ders.: Territorialstaat und Lehen. In: Studien zum mittelalterlichen Lehenswesen. Vorträge gehalten in Lindau am 10. -13. Oktober 1956. Vorträge und Forschungen 5. Lindau - Konstanz 1960, S. 195-228, hier: 228. Im Gegensatz dazu schrieb etwa Heinrich Mitteis dem Lehenswesen im Allgemeinen gar die führende Rolle im Prozess der Territorienbildung zu; siehe ders.: Lehnrecht und Staatsgewalt. Untersuchungen zur mittelalterlichen Verfassungsgeschichte. Weimar 1933, S. 450.

6 Die Lehenspolitik der Habsburger als Könige und Kaiser des römisch-deutschen Reiches und die Stellung der Herzoge von Österreich als Empfänger von Lehen müssen an dieser Stelle unberücksichtigt bleiben. Siehe dazu etwa Krieger, Karl-Friedrich: Die Lehnshoheit der deutschen Könige im Spätmittelalter (ca. 1200-1437). Untersuchungen zur deutschen Staats- und Rechtsgeschichte N.F. 23. Aalen 1979; Spreitzer, Renate: Die Belehnungs- und Bestätigungsurkunden König Sigismunds von 1421 für Herzog Albrecht V. von Österreich. Eine historische und textkritische Einordnung (1282-1729). Mitteilungen des Instituts für Österreichische Geschichtsforschung (= MIÖG) 114, 2006, S. 289-328.

7 Zur Zunahme von Schriftlichkeit im Lehenswesen des 13. Jahrhunderts siehe Spieß, Karl-Heinz: Formalisierte Autorität: Entwicklungen im Lehnsrecht des 13. Jahrhunderts. In: Autorität und Akzeptanz. Das Reich im Europa des 13. Jahrhunderts. Hg. von H. Seibert - W. Bomm - V. Türck. Ostfildern 2013, S. 137-147. Zu Mündlichkeit und Schriftlichkeit im Hoch- und Spätmittelalter siehe allgemein Andermann, Kurt: Pragmatische Schriftlichkeit. In: Höfe und Residenzen im spätmittelalterlichen Reich. Hof und Schrift. Hg. von W. Paravicini, bearb. von J. Hirschbiegel und J. Wettläufer. Residenzenforschung 15, 3. Ostfildern 2007, S. 37-60; Clanchy, Michael: From Memory to Written Record. England 1066-1307. Oxford 20123; Pragmatische Schriftlichkeit im Mittelalter. Erscheinungsformen und Entwicklungsstufen. Hg. von H. Keller - K. Grubmüller - N. Staubach. Münstersche Mittelalter-Schriften 65. München 1992; zur Situation im spätmittelalterlichen Österreich siehe Klebel, E.: Territorialstaat, S. 198; Weigl, Herwig: What to Write in Court. Literacy and Lawsuits in Late Medieval Austria. In: Charters and the Use of the Written Word in Medieval Society. Hg. von K. Heidecker. Utrecht Studies in Medieval Literacy. Turnhout 2000, S. 63-80. 
lehenrechtliche Verhältnisse gedeutet werden ${ }^{8}$ und fand in den habsburgischen Erblanden einen graduellen Niederschlag.

Im urkundlichen Bereich ist dieser zunächst in einer Zunahme von Lehenbriefen zu bemerken. Mit diesen vom Lehnsherrn über einen zuvor ausgeführten Belehnungsakt ausgestellten Urkunden wurden der Lehensempfänger, die Belehnung, die übertragenen Lehen sowie die sich daraus ergebenden Pflichten des Vasallen festgehalten. Derartige Lehenbriefe wurden von römisch-deutschen Königen und Kaisern seit der Mitte des 12. Jahrhundert zunächst vereinzelt, ab dem frühen 13. Jahrhundert in größerer Zahl ausgestellt. ${ }^{9}$ Im österreichischen Raum setzte diese Entwicklung, wie in anderen Fürstentümern und Grafschaften des Reiches, erst einige Jahrzehnte später ein. Der wohl älteste im Original überlieferte Lehenbrief eines österreichischen Herzogs wurde am 19. Mai 1232 in Oberitalien ausgestellt. ${ }^{10}$ Der Babenberger Friedrich II. belehnte (te infeudamus) als Herzog von Österreich und Steyr sowie als dominus Carniole ${ }^{11}$ in Pordenone den bereits unter seinem Vater tätigen Verwalter des babenbergisches Besitzes in Friaul, Ulrich Pitter aus Ragogna, in Ansehung seiner geleisteten Dienste mit Burghut und Maut zu Pordenone, quam nunc in tua tenes potestate. ${ }^{12}$

Eine größere Verbreitung von Lehenbriefen österreichischer Herzoge setzt, den allgemeinen Tendenzen in den Territorien des römisch-deutschen Reiches entsprechend, erst im Laufe des 14. Jahrhundert ein, wobei sich für diese Urkundenform bald ein relativ konstantes Formular entwickelte. Spätmittelalterliche Lehenbriefe werden - selbst im Falle umfangreicher Besitzübertragungen - zumeist in der Form einfacher Privilegien ausgestellt. Dementsprechend wird der landesfürstliche Aussteller in der Intitulatio mit

8 Siehe Spieß, K.-H.: Autorität, S. 146.

9 Schieffer, Rudolf: Das Lehnswesen in Königsurkunden von Lothar III. bis Friedrich I. In: Das Lehnswesen im Hochmittelalter. Forschungskonstrukte - Quellenbefunde - Deutungsrelevanz. Hg. von J. Dendorfer R. Deutinger. Mittelalter-Forschungen 34. Ostfildern 2010, S. 79-90, hier: 82; ders.: Das Lehnswesen in den Urkunden der Kaiserin Konstanze, in den frühen Königsurkunden Friedrichs II. und in den Urkunden der Könige von Jerusalem. In: Ausbildung und Verbreitung des Lehnswesens im Reich und in Italien im 12. und 13. Jahrhundert. Hg. von K.-H. Spieß. Vorträge und Forschungen 76. Ostfildern 2013, S. 221-238, hier: 230234. Zur Verbreitung von Lehenbriefen außerhalb des deutschen Sprachraumes sowie dem Privilegium minus als früher Lehenbrief siehe Maleczek, Werner: Das Privilegium minus. Diplomatische Gesichtspunkte. In: Die Geburt Österreichs - 850 Jahre Privilegium minus. Hg. von P. Schmid - H. Wanderwitz. Regensburger Kulturleben 4. Regensburg 2007, S. 103-141, hier: 131-139.

10 Urkundenbuch zur Geschichte der Babenberger in Österreich. Band 2: Die Siegelurkunden der Babenberger und ihrer Nachkommen von 1216-1279. Bearb. von H. Fichtenau und E. Zöllner. Publikationen des Instituts für Österreichische Geschichtsforschung III/2. Wien 1954, Nr. 299. Zu den etwa gleichzeitig auftretenden ältesten Lehensurkunden in der Pfalzgrafschaft bei Rhein sowie der Grafschaft Katzenelnbogen siehe Spieß, K.-H.: Lehnsrecht und Diestelkamp, B.: Lehnrecht.

$11 \mathrm{Zu}$ Friedrichs Übernahme des Titels „Herr von Krain“ siehe Ficker, Adolf: Herzog Friedrich II., der letzte Babenberger. Innsbruck 1884, S. 169-178.

12 Ältere Erwähnungen von beneficia und feuda in österreichischen Herzogsurkunden stehen zumeist mit der herzoglichen Bestätigung von auf Lehensgut bezogenen Verfügungen, wie Verkäufen, Schenkungen und Stiftungen von Lehensgut durch Vasallen, in Zusammenhang; siehe etwa Urkundenbuch zur Geschichte der Babenberger in Österreich. Band 1: Die Siegelurkunden der Babenberger bis 1215. Vorbereitet von O. Frh. von Mitis, bearb. von H. Fichtenau und E. Zöllner. Publikationen des Instituts für Österreichische Geschichtsforschung III/I. Wien 1950, Nr. 59 (Wien, 1181 X 23), 184 (Passau, 1212 V 13) und 186 (Marburg, 1213). 
seinem einfachen Titel geführt, auch auf eine klassische Arenga wird üblicherweise verzichtet. Stattdessen wird der Kontext mit einer knappen Publicatio eingeleitet, im 14. Jahrhundert häufig mit den Worten tun kunt bzw. bekennen und tun kunt; ab dem späten 14. Jahrhundert setzt sich das einfache bekennen durch, gelegentlich in der erweiterten Form: bekennen offentlich mit disem brief. ${ }^{13}$ Den Beginn der Dispositio leitet häufig die Bitte des Empfängers um Belehnung bzw. die Intervention eines Dritten zugunsten des Belehnten ein ${ }^{14}$, deren Gewährung der Urkundenaussteller zuweilen mit geleisteten bzw. noch zu erbringenden Verdiensten des Belehnten begründet ${ }^{15}$. Wenn der Petent weder konkrete Dienstleistungen vorweisen noch entsprechende Rechtsansprüche geltend machen konnte, wurde zuweilen eine Verleihung von sundern gnaden vorgenommen. ${ }^{16}$

Die eigentliche Investitur wird zumeist in doppelter Weise angegeben, nämlich als Ausdruck der eigentlich Rechtshandlung (haben wir verlihen), ergänzt um eine Bestätigung, die den dinglichen Aspekt der Urkunde als Beweismittel betont (und leihen auch mit dem brief oder craft dis briefs). Darüber hinaus finden besonders seit dem 15. Jahrhundert im Bereich der landesfürstlichen Lehenbriefe aus dem kanonischen Recht übernommene Formeln wie wissentlich/ex certa scientia bzw. der Rechtsvorbehalt was wir im von rechts wegen verleihen sullen und mugen Verwendung. ${ }^{17}$

13 Siehe dazu auch Lackner, Christian: Hof und Herrschaft. Rat, Kanzlei und Regierung der österreichischen Herzoge (1365-1406). MIÖG, Ergänzungsband 41. Wien - München 2002, S. 247f.

14 Siehe etwa Haus-, Hof- und Staatsarchiv (= HHStA) Wien, Allgemeine Urkundenreihe (= AUR) 1467 II 28 : bekennen, das für uns komen ist unser getrewr Sigmund Kirichperger und bat uns diemutigkleich, das wir im [...] geruchten zu verleihen bzw. ebd. 1477 II 26: bekennen, das uns unser getrewer Osterman Awrsperger funf huben [...] aufgesannd und diemuticlich gebeten hat, die unserm getrewn Niclasen Durrer zu verleyhen, wann er im die verkauft hiet.

15 So belehnte Kaiser Friedrich III. den Hauptmann von Pordenone, Phebusch von Turn, und den Krainer Ritter Friedrich von Ungersbach aufgrund ihrer geleisteten Dienste bey uns in unser burckh zu Wienn, als wir darinn von hern Albrechten von Österreich, ettlichen lanndtleuten und den von Wienn behawrt und belagert gewesen sein; siehe HHStA Wien, AUR 1463 I 1.

16 Siehe Regesta Habsburgica. Regesten der Grafen von Habsburg und der Herzoge von Österreich aus dem Hause Habsburg V-2. Bearb. von C. Lackner unter Mitarbeit von C. Feller und S. Seitschek. Wien - München 2010, etwa Nr. 1155 und 1209. Bernhard Diestelkamp und Karl-Heinz Spieß bezeichnen diese als Vermittler der Maximen politischen Handelns zu interpretierenden Formeln als „knappe Dienst- bzw. Gnadenarengen“ im Sinne Heinrich Fichtenaus; siehe Diestelkamp, B.: Lehnrecht, S. $51-57$ und Spieß, K.-H.: Lehnsrecht, S. 37-41, bes. S. 41. Nach Fichtenau sei die im spätmittelalterlichen Urkundenwesen häufig festzustellende Praxis, Arengen hinter die Publicatio zu stellen, „eine aus dem Westen importierte Mode“; siehe Fichtenau, Heinrich: Arenga. Spätantike und Mittelalter im Spiegel von Urkundenformeln. MIÖG Ergänzungsband 18. Graz - Köln 1957, S. 164. Zu Arengen in der habsburgischen Kanzlei des 14. Jahrhunderts siehe Lackner, C.: Hof, S. 247.

17 Siehe dazu allgemein die Studien von Othmar Hageneder, insbesondere: ders.: Probleme des päpstlichen Kirchenregiments im hohen Mittelalter (Ex certa scientia, non obstante, Registerführung). Lectiones eruditorum extraneorum in facultate philosophica Universitatis Carolinae Pragensis factae 4, 1995, S. 49-77; ders.: Kanonisches Recht, Papsturkunde und Herrscherurkunde. Überlegungen zu einer vergleichenden Diplomatik am Beispiel der Urkunden Friedrichs III. Archiv für Diplomatik 42, 1996, S. 419-443. Vgl. auch Isenmann, Eberhard: Reichsrecht und Reichsverfassung in Konsilien reichsstädtischer Juristen (15.-17. Jahrhundert). In: Die Rolle der Juristen bei der Entstehung des modernen Staates. Hg. von R. Schnur. Berlin 1986, S. 545-628, bes. 568f., und Wild, Joachim: Schriftlichkeit in der Verwaltung am Beispiel der Lehenbücher in Bayern. In: Schriftlichkeit und Lebenspraxis im Mittelalter. Erfassen, Bewahren, Verändern. Hg. von H. Keller C. Meier - T. Scharff. Münstersche Mittelalter-Schriften 76. München 1999, S. 69-77, hier: 71. 
Die Auflistung der übertragenen Lehengüter kann vor oder nach der Investiturformel erfolgen, als geographische Ordnungskriterien werden zumeist Länder, Herrschaften und Pfarren herangezogen. Zuweilen wurde bei der Abfassung eines Lehenbriefes in der landesfürstlichen Kanzlei auf den Inhalt einer oder mehrerer Vorurkunden zurückgegriffen, neu hinzugekommene Lehengüter wurden dabei ohne Beachtung vorhergehender Ordnungskriterien am Ende des Lehenverzeichnisses eingefügt. ${ }^{18}$

An diese Auflistung schließt üblicherweise eine Gewährleistungsformel mit näheren Angaben zur Rechtsform der Lehensübertragung. Zumeist begnügte man sich in der habsburgischen Kanzlei mit der Angabe, der Belehnte möge die ihm übertragenen Besitzungen nach Lehens- und Landrecht innehaben. ${ }^{19}$ Ab dem 15. Jahrhundert werden häufig auch die zu erwartenden Leistungen des Lehensmannes genannt, zumeist in Form einer allgemeinen Forderung, wonach sich der Belehnte zukünftig gehorsam und dienstbereit zu zeigen habe, als lehenslewt irem lehensherren phlichtig sint und gepunden. ${ }^{20}$ In seltenen Fällen werden auch näher erläuterte Leistungen des Lehensmannes wie die Offenhaltung einer übertragenen Burg ${ }^{21}$ oder die Abwicklung eines für den Lehensherrn günstigen Tauschgeschäftes gefordert. ${ }^{22}$ Häufig findet in diesen Urkunden auch der geforderte Lehenseid Erwähnung, etwa im Rahmen einer Bestätigung über einen bereits geleisteten oder einer obligatorischen Bestimmung über einen ausstehenden Lehenseid. ${ }^{23}$

Sanctiones bzw. Pönformeln finden in Lehensurkunden bemerkenswerterweise in der Regel keine Verwendung ${ }^{24}$ und kommen lediglich in jenen Diplomen vor, die neben lehenrechtlichen Besitzübertragungen noch die Verleihung oder Bestätigung weiterer Privilegien beinhalten. In diesen Fällen erfolgt oft die Anweisung, unter Androhung von Geldstrafe, Ungnade oder Huldverlust keinen Eingriff in die Rechte des Begünstigten zu tätigen. ${ }^{25}$

Die übrigen Formularteile der Lehenbriefe entsprechen den Gewohnheiten der spätmittelalterlichen habsburgischen Kanzleien. Dies gilt auch für die äußeren Merkmale;

18 Siehe etwa Regesten Kaiser Friedrichs III. (1440-1493) nach Archiven und Bibliotheken geordnet. Heft 22. Hg. von H. Koller, P.-J. Heinig und A. Niederstätter, bearb. von C. Ottner. Wien u. a. 2007, Nr. 167 und 284.

19 Siehe dazu Härtel, Reinhard: Neue Wege zur Erschließung von Urkundenformeln. Lectiones eruditorum extraneorum in facultate philosophica Universitatis Carolinae Pragensis factae 4, 1995, S. 7-45.

20 Siehe etwa den Lehenbrief Herzog Friedrichs IV. von Österreich für Michael von Wolkenstein und dessen Brüder und Vettern Oswald, Leonhard, Konrad und Veit über Burg und Gericht Wolkenstein vom 8. September 1422: Die Lebenszeugnisse Oswalds von Wolkenstein. Edition und Kommentar. Band 2: 1420-1428. Hg. von A. Schwab. Wien u.a. 2001, Nr. 133.

21 Siehe ebd.

22 Regesta Habsburgica III. Bearb. von L. Gross. Innsbruck 1922, Nr. 740.

23 Regesten Kaiser Friedrichs III. Heft 13. Hg. von H. Koller, P.-J. Heinig und A. Niederstätter, bearb. von P. Herold und K. Holzner-Tobisch. Wien u.a. 2001, Nr. 164; ebd. Heft 19. Hg. von H. Koller, P.-J. Heinig und A. Niederstätter, bearb. von S. Dünnebeil und P. Herold. Wien u.a. 2004, Nr. 329.

24 Lackner, C.: Hof S. 250. Siehe auch Rübsamen, Dieter: Buße und Strafe. Zu den Pönformeln spätmittelalterlicher Königsurkunden besonders unter Friedrich III. In: Ex Ipsis Rerum Documentis. Festschrift für H. Zimmermann zum 65. Geburtstag. Hg. von K. Herbers - H.-H. Kortüm - C. Servatius. Sigmaringen 1991, S. 117-133, hier 122 . 
bei Lehenbriefen handelt es sich zumeist um einfache Privilegien auf Pergament mit Hängesiegel. ${ }^{26}$

Lehenbriefe beleuchten in der Regel nur einen Teilbereich des spätmittelalterlichen Lehenswesens, denn sie betreffen überwiegend den sogenannten Mannfall, d.h. den zumeist todesbedingten Ausfall des Vasallen. Darüber hinaus werden in Urkunden anfangs in erster Linie lehenrechtliche Unregelmäßigkeiten und Ausnahmen behandelt, deren urkundliche Bestätigung vor allem im Interesse der Lehensempfänger lag und daher auch von diesen erbeten, bezahlt und verwahrt wurde. ${ }^{27}$

Wie aus einem Taxregister der Kanzlei Kaiser Friedrichs III. für die Jahre 1471-75 hervorgeht wird, stellten Lehenbriefe für den Herrscher eine beträchtliche Einnahmequelle dar. ${ }^{28}$ Die Taxe richtete sich in erster Linie nach Finanzkraft und Stellung des Petenten und dem Umfang der übertragenen Lehen. So erhielten zahlreiche Vasallen ihre Lehenbriefe kostenlos oder zu einer vergleichsweise günstigen Taxe von einem oder zwei Gulden, quia pauper rusticus ${ }^{29}$, aufgrund des geringen Umfanges der empfangenen Besitzungen $^{30}$, der Jugend des Vasallen ${ }^{31}$ oder in Ansehung geleisteter Dienste. ${ }^{32}$ Vermögende Lehensträger hatten für ihre Urkunden durchaus mehrere Dutzend Gulden zu bezahlen ${ }^{33}$ oder übergaben dem Kaiser als Gegenleistung kostbare Geschenke wie einen Saphirring aus ehemals herzoglichem Besitz ${ }^{34}$ bzw. eine Schiffsminiatur aus purem

26 Zuweilen wird wohl aus Kostengründen anstelle des an einer Pergamentpressel anhangenden kaiserlichen Siegels ein rückseitig aufgedrücktes Siegel angebracht; siehe etwa Regesten Kaiser Friedrichs III. Sonderband 2. Das Taxregister der römischen Kanzlei 1471-1475. Hg. von H. Koller, P.-J. Heinig und A. Niederstätter, bearb. von P.-J. Heinig und I. Grund. Wien u.a. 2001, Nr. 987, 1337, 2259, 2887, 3837f., 3840 (sub sigillo appresso, eyn armen gebueren), 3856 (sub sigillo appresso fur eynen gebueren), 3908, 3915, 3918, 3981; ebd. Heft 18. Hg. von H. Koller, P.-J. Heinig und A. Niederstätter, bearb. von S. Dünnebeil und P. Herold. Wien u.a. 2004, Nr. 105. Der kaiserliche Rat und Gesandte Rudolf von Sulz erhielt hingegen - wohl aufgrund seiner geleisteten Dienste - kostenlos einen mit dem kaiserlichen Majestätssiegel beglaubigten Lehenbrief, nachdem er in der Kanzlei eine entsprechende Vorurkunde sub parvo sigillo vorlegte und retournierte; siehe Regesten Kaiser Friedrichs III., Sonderband 2, N.F.129.

27 Siehe Spieß, K.-H.: Autorität, S. 140; ders.: Lehnsbrief. In: Handwörterbuch zur Rechtsgeschichte, Bd. 2. Berlin 1977, Sp. 1701f.

28 Siehe Regesten Kaiser Friedrichs III., Sonderband 2. In diesem Taxregister wurden an die römische Kanzlei geleistete Taxen verzeichnet. Es betrifft daher in erster Linie Verleihungen von Reichslehen, allerdings muss für die erbländische Kanzlei Friedrichs III. nach Ausweis der zahlreich überlieferten landesfürstlichen Kanzleiregister mit einem noch größeren Anteil ausgestellter Lehenbriefe gerechnet werden.

29 Ebd., Nr. 77, 188, 208f., 541, 559, 614, 616, 729 (quia omnino erat pauper, prout socii in cancellaria unamecum clare vidimus), 1635 (eyn armer gebuer), 2887 (fur eynen armen man; ex quo pauper fuit, ideo dedit 1 fl.), 3840 , 3856, 3969 (dominus imperator scripsit pro eo manu propria, ideo dominus dedit gratis pro deo, quia pauper).

30 Ebd., Nr. 1030 (parvum feodum), 1814 (sint slechtte zinse), 2994 (das lehen ist cleyn), 3982 (eyns cleynen gutlins halben).

31 Ebd., Nr. 837.

32 Ebd., Nr. 3072: dominus dedit gratis attentis serviciis, qua sepius fecit domino.

33 Siehe etwa ebd., Nr. 117, 184, 325, 2882.

34 Ebd., Nr. 2767. 
Silber. ${ }^{35}$ Über die Höhe der zu leistenden Taxen wurden überdies Verhandlungen zwischen dem Lehensherrn und seinen Vasallen geführt. ${ }^{36}$

Eine erhöhte Taxe hatte der Lehensträger auch dann zu leisten, wenn die persönliche Leistung des Lehenseides vor dem Landesfürsten aus diversen Gründen nicht möglich war. In diesen Fällen konnte der Lehensherr einen Dritten kommissarisch mit dem Empfang der Eidesleistung beauftragen. ${ }^{37}$ Solche Kommissare in Lehenssachen wurden von den habsburgischen Herzogen insbesondere bei größerer räumlicher Entfernung des Lehengutes zuweilen auch dauerhaft eingesetzt. So erteilte etwa Herzog Albrecht III. 1367/68 dem habsburgischen Landvogt im Elsass, Sundgau und Breisgau die Erlaubnis, kleinere landesfürstliche Lehen bis zu einem Wert von 5 Mark Geldes eigenmächtig zu verleihen und auch deren Veräußerung zu gestatten. ${ }^{38}$

Während der persönliche Belehnungsvorgang in den Hintergrund trat ${ }^{39}$ und teilweise von Stellvertretern vorgenommen wurde, erhielten Lehenbriefe zunehmend die Funktion eines zentralen Beweismittels für eine gewünschte Lehenserneuerung. ${ }^{40}$ Wie in anderen Bereichen des spätmittelalterlichen Urkundenwesens trat jedoch auch hier ein gewichtiger Mangel der landesfürstlichen Kanzleigebarung zu Tage. Im Jahr 1484 wurde etwa im Zuge eines Rechtsstreits zwischen dem Kloster Lambach und Jörg Kirchberger um ein Fischwasser vor dem Landeshauptmann ob der Enns die Rechtskraft landesfürstlicher Lehnbriefe im Allgemeinen relativiert. So brachte der Lambacher Abt als Kläger vor, dass der Beklagte zweifellos auf dessen Bitte hin vom Landesfürsten mit einem bestimmten Fischwasser belehnt worden sei, denn es käme häufig vor, dass jemand zu einem Herrn käme und Lehen anzeige. So mechte der herr nichtz daran verlieren und lihe nit allain ain(em), sondern mocht yedem, der des begerte, bis in die neund hand, was er zu recht daran verleihen solt, doch yederman an sein(em) rechten unschedlich. In dieser Weise sei dem Beklagten das Fischwasser verliehen worden, doch könne - so der Abt weiter ein solich [...] blosse unbewerte wort kain nutz bringen und damit den Rechten des Klosters nicht schaden. ${ }^{41}$ Die Beweiskraft eines Lehenbriefes wurde vom Kläger mit Hinweis auf

35 Ebd., Nr. $3125 f$.

36 Ebd., Nr. 2602, 2704, 3344.

37 Siehe etwa ebd., Nr. 893, 1993, 2089, 2155, 2569, 2586f., 2769, 2870, 3340, 3425, 3874, 3983, 4048, F33.

38 Regesta Habsburgica V-1. Bearb. von Christian Lackner unter Mitarbeit von Claudia Feller. Wien - München 2007, Nr. 219. Zu einer vergleichbaren Kommission unter Herzog Leopold III. siehe ebd. V-2, Nr. 1068 (1374 I 6).

39 In einem Schreiben vom 13. Oktober 1460 forderte Kaiser Friedrichs III. seinen böhmischen Söldnerführer Jan Witowetz zu Seger, Inhaber der Feste Lienz, auf, neben der bereits erfolgten schriftlichen Übertragung auch eine förmliche und persönliche Belehnung, als sich gepüret, durch den Erzbischof von Salzburg als Lehensherrn anzustreben. Uns bedunkcht auch solhs gut und für dich ze sein, wan dadurch dein gerechtikait besterkcht wirdet; siehe Chmel, Joseph: Regesta chronologico-diplomatica Friderici IV. Romanorum regis [...]. ND Hildesheim 1962, Nr. 3832.

40 Am Kammergericht Kaiser Friedrichs III. wurden Lehenbriefe als Beweismittel vorgebracht und verlesen; siehe etwa Die Protokoll- und Urteilsbücher des Königlichen Kammergerichts aus den Jahren 1465 bis 1480, Band 1. Hg. von F. Battenberg und B. Diestelkamp, bearb. von C. Magin und J. Maurer. Quellen und Forschungen zur höchsten Gerichtsbarkeit im alten Reich 44. Köln u.a. 2004, S. 218f.; Regesten Kaiser Friedrichs III. Heft 3. Hg. von H. Koller, bearb. von P.-J. Heinig. Wien u.a. 1983, Nr. 49.

41 Stiftsarchiv Lambach, Urkunde Nr. 1864. Siehe dazu Hageneder, O.: Recht, S. 430. 
den bereits oben erwähnten Rechtsvorbehalt was wir zu recht daran verleihen sollen in Frage gestellt, da am landesfürstlichen Hof die Angaben von Petenten über die ihnen zustehenden Lehen häufig urkundlich bestätigt worden seien, ohne diese näher zu überprüfen.

Demgegenüber stehen jedoch einige Quellen, die diese Sorglosigkeit bzw. fehlenden Kontrollmöglichkeiten von Seiten der landesfürstlichen Kanzlei relativieren. So setzte etwa bereits Herzog Leopold im Jahr 1326 zur Klärung der Streitfrage, ob bestimmte Güter zu Ettiswil habsburgische Lehen oder freies Eigen der nahen Zisterze St. Urban seien, eine Kommission mit dem Auftrag ein, vor Ort Zeugenaussagen der ansässigen Untertanen über ihre rechtliche Zugehörigkeit unter Eid aufnehmen zu lassen. Nach deren Aussagen verzichtete der Landesfürst schließlich auf seine lehenrechtlichen Ansprüche. $^{42}$

Im Jahr 1370 habe Herzog Leopold III. mit gutem urkunde und versigelten briefen bewiesen, dass Burg und Stadt Engen sowie Burg Hewenegg landesfürstliche Lehen seien, woraufhin der bisherige Inhaber um Belehnung angesucht habe. ${ }^{43}$

Urkundliche Belege wurden zuweilen auch von der landesfürstlichen Verwaltung als Bedingung für eine Belehnung eingefordert. So enthält eine am 24. April 1479 am Hof Friedrichs III. eingereichte schriftliche Supplik mit der Bitte um Belehnung mit ererbten Gütern und Gülten in der Steiermark den rückseitigen Kanzleivermerk: sofer er die lehenbrif pringt, sol man im lehen. ${ }^{44}$ Konnten Petenten in der Kanzlei keine entsprechenden Vorurkunden übermitteltn, wird in den Privilegien zuweilen darauf hingewiesen. ${ }^{45}$

Eine weitere für die Untersuchung des Lehenswesens in den habsburgischen Erblanden zentrale Quellengruppe stellen die in der landesfürstlichen Kanzlei geführten Lehenbücher dar, deren Inhalt und Charakteristika im Rahmen dieses Beitrages nur knapp umrissen werden können. ${ }^{46}$

42 Regesta Habsburgica V-2, Nr. 1702.

43 Ebd. $V-1$, Nr. 550.

44 HHStA Wien, AUR 1479 IV 24.

45 Siehe etwa Regesten Kaiser Friedrichs III. Heft 22, Nr. 167: wann [...] im die lehenbrieff, so er vormals darumb von unsern vorvarn fursten von Österreich gehabt hiete, verprunnen wern. Nach dem Verlust eines Lehenbriefes konnte eine neuerliche Urkundenausstellung erbeten werden: wann [...] im die brief, so er deshalben gehabt hiet, durch die Turkhen von hannden komen wern; siehe Regesten Kaiser Friedrichs III. Heft 30. Hg. von P.-J. Heinig, C. Lackner und A. Niederstätter, bearb. von P. Gretzel. Wien u.a. 2014, Nr. 91.

46 Siehe allgemein Lippert, Woldemar: Die deutschen Lehnbücher. Beitrag zum Registerwesen und Lehnrecht des Mittelalters. Leipzig 1903, bes. S. 147, 160-162 und 173 (Rezension: Lechner, Johann: MIÖG 27, 1906, S. 505-511; Spieß, Karl-Heinz: Lehnbuch, Lehnregister. In: Handwörterbuch zur Rechtsgeschichte, Bd. 2. Berlin 1977, Sp. 1686-88. Zur Überlieferung in den habsburgischen Erblanden siehe Seeliger, Gerhard: Die Registerführung am deutschen Königshof bis 1493. MIÖG Ergänzungsband 3, 1890/94, S. 223-363, hier: 302-310; Stowasser, Otto H.: Die österreichischen Kanzleibücher vornehmlich des 14. Jahrhunderts und das Aufkommen der Kanzleivermerke. MIÖG 35, 1914, S. 688-724. Zu einzelnen habsburgischen Lehenbüchern siehe Tepperberg, Christoph: Das Lehenbuch Herzog Albrechts III. von 1380-1394. Unsere Heimat N.F. 48, 1977, S. 221-233; Mühlberger, Kurt: Das ältere Lehenbuch Herzog Albrechts V. von Österreich 1411-1418. Unpubl. Prüfungsarbeit am Institut für Österreichische Geschichtsforschung. Wien 1983. Zur Überlieferungssituaton in Bayern siehe Wild, J.: Schriftlichkeit. 
Während Lehenbriefe in der Regel mit dem Ausfall des Vasallen (Mannfall) in Zusammenhang stehen, wird die Anlage von Lehenbüchern in den habsburgischen Erblanden zumeist mit dem todesbedingten oder infolge von Herrschaftsteilungen eintretenden Verlust des Lehensherrn (Herrenfall) begründet. Häufig wurden diese Lehenbücher jedoch in späteren Jahren auch zur Verzeichnung von Belehnungen nach einem Mannfall weiter verwendet. ${ }^{47}$ Einträge zu Lehensübertragungen sind darüber hinaus auch in Urbaren, Traditionsbüchern und allgemeinen Kanzleiregistern zu finden. ${ }^{48}$

Im Herzogtum Österreich stammt das älteste Lehenbuch aus dem Jahr $1380^{49}$, Auslöser für dessen Anlage war der Neuberger Vertrag des Jahres 1379, der zu einer Herrschaftsteilung der Brüder Albrecht und Leopold führte. Da Herzog Albrecht III. nunmehr alleiniger Lehensherr in Österreich ob und unter der Enns war, wurde eine sogenannte Neurufung der österreichischen Lehen im Rahmen eines Lehen(hof)tages notwendig. ${ }^{50}$

Die habsburgischen Lehenbücher des 14. und 15. Jahrhundert enthalten zahlreiche Hinweise über den Ablauf eines Lehentages. So ist in einem Lehenbuch des Ladislaus Postumus abschriftlich ein sogenannter beruffzedel aus Jahr 1455 zusammen mit einem landesfürstlichen Mandat überliefert, in dem allen Richtern und Räten der österreichischen Städte und Märkte befohlen wird, den Text des übersandten Zettels an den jeweiligen Wochenmärkten öffentlich ausrufen zu lassen. ${ }^{51}$ Der Text dieser Verlautbarung lautet: Hort und lost, hort, lost. Ew tut unser gnedigister herr kunig Lasslaw zu wissen, daz sein kunigelich gnad alle die lehen seins furstentums Österreich niderhalb und ob der Enns, die von seinen kuniglichen gnaden zu lehen geent, auf sand Jorgen tag schiristkunftigen [24. April] leihen will oder emphelhen zu leihen, als solher lehen und lannds recht ist.

Der Ort dieses Lehentages wird nicht genannt, unter Herzog Rudolfs IV. fand dieser auf dem Platz am Hof in Wien statt, und noch ein Jahrhundert später wurde im Vorfeld dieses Ereignisses auf Kosten der Stadt Wien auff dem hoff von Zimmerern ein hölzerner Lehenstuhl für den neuen österreichischen Landesfürsten Kaiser Friedrich III. angefertigt. $^{52}$

Im Zuge eines solchen Lehentages hatten die Lehensempfänger schriftliche Lehenbekenntnisse einzureichen, die in den Quellen als Zettel bzw. cedulae bezeichnet werden und das jeweilige Lehengut zum Inhalt hatten. In der landesfürstlichen Kanzlei wurden

47 Bei Eintreten des Mannfalles hatte der Erbe den Lehensherrn binnen eines Jahres um Belehnung zu ersuchen. Zur Mutungsfrist in den habsburgischen Erblanden siehe Mühlberger, K.: Lehenbuch, S. 18.

48 Siehe Lackner, Christian: Studien zum ältesten allgemeinen Register der österreichischen Herzogskanzlei. MIÖG 100, 1992, S. 237-253, hier: 242. Spieß, K.-H.: Lehnbuch, Sp. 1687, und Lechner, Johann: Ein unbeachtetes Register König Friedrichs IV. (III.) 1440-1442. MIÖG 20, 1899, S. 52-68, hier: 54.

49 Zu zwei älteren, den habsburgischen Lehenhof in den Vorlanden betreffenden Lehenbüchern Albrechts II. und Rudolfs IV. siehe Mühlberger, K.: Lehenbuch, S. 6f.

50 Siehe Tepperberg, C.: Lehenbuch, S. 221.

51 HHStA Wien, Hs. B 25, f. 7v. Zum Folgenden siehe auch Tepperberg, C.: Lehenbuch, S. 222-226.

52 Ebd., S. $223 f$. 
diese von den Vasallen eingereichten Zettel gesammelt und in die Lehenbücher übertragen. ${ }^{53}$

Die Lehenbekenntnisse enthalten eine mehr oder weniger umfangreiche Auflistung des anzeigten Lehenbesitzes, eingeleitet zumeist mit der Wendung: hie ist vermerkt, was ich N. von meinem gnadigen herren zu lehen hab: [...]. Bei der Übertragung in die Lehenbücher wurden diese subjektiv formulierten Angaben von den Schreibern der landesfürstlichen Kanzlei in den meisten Fällen objektiviert, zumeist in der Form: item N. hat ze lehen [...]. Zuweilen ist es jedoch bei diesem Vorgang zu Ungereimtheiten gekommen. So findet man insbesondere in älteren habsburgischen Lehenbüchern häufig Einträge, die zwar die einzelnen Lehengüter beinhalten, nicht aber den Namen des Lehenträgers, da dieser auf den eingereichten Lehenbekenntnissen nicht verzeichnet gewesen sei ${ }^{54}$ Auch konnten einige dieser Zettel aufgrund sprachlicher Probleme nicht in das Lehenbuch übernommen werden. Stattdessen merkte ein Kanzleischreiber an, unter jenen in einem Sack aufbewahrten Zetteln mit Lehenbekenntnissen befänden sich zwei, die er nicht lesen könne, propter innotitiam loquele, quae Gallica..$^{55}$ Darüber hinaus gaben einige Vasallen an, nicht in der Lage zu sein, ihren vollständigen Lehensbesitz anzuführen ${ }^{56}$, oder legten der Kanzlei anstelle von Zetteln ältere Lehenbriefe vor. ${ }^{57}$

Nach Christoph Tepperberg hätten Lehenbücher lediglich als Hilfsmittel der landesfürstlichen Kanzlei gedient und seien dem Wunsch nach besserer Übersicht des herzoglichen Besitzes entsprungen. ${ }^{58}$ Für diese These spricht auch die Tatsache, dass Lehenbücher im Gegensatz zu Lehensurkunden in keinem der zahlreich überlieferten Gerichtsprozesse des 15. Jahrhunderts um den rechtmäßigen Besitz von Lehengut als Beweismittel herangezogen wurden. Lehenbücher waren hinsichtlich ihrer Beweiskraft nur in Ausnahmefällen urkundlichen Quellen gleichgestellt, wie ein Privileg Friedrichs III. für Erzbischof Jakob von Trier vom 27. August 1442 zeigt. Darin heißt es, Erzbischof Jakob habe dem römisch-deutschen König persönlich vorgebracht, dass im Zuge der sogenannten Trierer Stiftsfehde erzbischöfliche Lehen nicht ordnungsgemäß verliehen worden und viele Lehenbriefe in falsche Hände gelangt seien. Deshalb befürchte Jakob, die erzbischöflichen Vasallen könnten ihre Lehen verschweigen, was zu einer Minderung seines Lehenhofes führen würde. Da jedoch sämtliche Lehen mit ihren rechtmäßigen Inhabern in den lehenpuchern, die man mannepucher nennet, verzeichnet seien, verfügt der König mit dieser Urkunde von sundern gnaden, dass den Einträgen in den erzbischöflichen

53 Lippert, W.: Lehnbücher, S. 35-37, 56-65; Tepperberg, C.: Lehenbuch, S. 224f; Mühlberger, K.: Lehenbuch, S. 52f. Vereinzelt sind auch im Original erhaltene Lehenzettel überliefert; siehe etwa Niederösterreichisches Landesarchiv St. Pölten, Lehenregister C (1446); HHStA Wien, Hs. W 722, f. 84.

54 Siehe etwa HHStA Wien, Hs. W 722, f. 140v: nomen conductoris non fuit cedulae insertum.

55 Tepperberg, C.: Lehenbuch, S. 225.

56 Ebd., S. 224: was er furbaz ervert, daz will er auch ze wissen tun und emphahen.

57 Mühlberger, K.: Lehenbuch, S. 53f.

58 Tepperberg, C.: Lehenbuch, S. 227. 
Lehenbücher im Falle des Verlusts der entsprechenden Lehenbriefe durch Krieg und unrate dieselbe Glaubwürdigkeit wie den Urkunden selbst zukommen soll. ${ }^{59}$

Wie ein Eintrag in einem Lehenbuch Albrechts V. belegt, scheint den Einträgen in Lehenbüchern auch hinsichtlich der Mutungsfrist nach dem Mannfall eine rechtliche Relevanz zugekommen zu sein. In diesen Fällen schrieb der habsburgische Lehensherr den rechtmäßigen Erben vor, dacz si uns dieselben lehen inner jarsfrist mit namen in unser lehenpuch geschriben geben. ${ }^{60}$

Abgesehen von Lehenbriefen und -büchern sind noch zahlreiche weitere schriftliche Quellen zum Lehenswesen in den habsburgischen Erblanden überliefert, so etwa die von Vasallen als Gegenstücke zu den landesfürstlichen Lehenbriefen ausgestellten Lehenreverse $^{61}$ oder Interimsbescheinigungen in Form von Indultbriefen, die dem Vasallen eine erweiterte Frist zur Lehensmutung gewährten. ${ }^{62}$ Eine weitere Gruppe von Urkunden steht mit der Veräußerung von Lehen durch den Lehensmann im Zusammenhang. Hier wären etwa landesfürstliche Bestätigungen für Verkäufe, Verpfändungen oder Stiftungen von Lehengut zu nennen ${ }^{63}$, denn dieses blieb auch nach einer Belehnung Eigengut des Lehensherrn, weshalb eine Veräußerung von Lehen durch die Vasallen der ausdrücklichen Zustimmung des Herrn bedurfte. ${ }^{64}$

In einem Register der erbländischen Kanzlei Kaiser Friedrichs III. finden sich unter dem Titel Vermerkcht, wie es all fursten im reich gemainkleich mit irn lehen gen irn lehensmann halten lehenrechtliche Ausführungen, die großteils von der Hand des kaiserlichen Protonotars und Juristen Johannes Rehwein ${ }^{65}$ stammen und neben rechtlichen Bestimmungen über ledige bzw. heimgefallene Lehen auch die Position des habsburgischen Landesfürsten zum Lehensverkauf zusammenfassen. ${ }^{66}$ Demnach stehe dem landesfürstlichen

59 Siehe Regesten Kaiser Friedrichs III. Heft 9. Hg. von H. Koller und P.-J. Heinig, bearb. von R. Neumann. Wien u.a. 1996, Nr. 56.

60 HHStA Wien, Hs. B 21, f. 68r. Siehe auch Mühlberger, K.: Lehenbuch, S. 18f.

61 Vgl. etwa den Lehenrevers von Konrad von Wittenheim, Bürger zu Colmar, vom 30. Jänner 1315 an den österreichischen Herzog Leopold über Rechte in Heitersheim im Breisgau: Regesta Habsburgica III, Nr. 84.

62 Zur Mutungspflicht der Vasallen siehe Mitteis, H.: Lehnrecht, S. 638-642; Diestelkamp, B.: Lehnrecht, S. 140f.; Spieß, K.-H.: Lehnsrecht, S. 101-103.

63 Siehe etwa Regesta Habsburgica II-1. Bearb. von H. Steinacker. Innsbruck 1905, Nr. 146, 148, 269; ebd. III, Nr. 1365, 1409, 1691, 1750, 1940.

64 Siehe dazu Mitteis, H.: Lehnrecht, S. 629-638; Diestelkamp, B.: Lehnrecht, S. 154f.; Spieß, K.-H.: Lehnsrecht, S. 104-111.

65 Zu Johannes Rehwein siehe Luger, Daniel: Humanismus und humanistische Schrift in der Kanzlei Kaiser Friedrichs III. (1440-1493). MIÖG Ergänzungsband 60, 2016, S. 97-110 (mit weiterer Literatur).

$66 \mathrm{Zu}$ den vor allem auf dem Schwabenspiegel beruhenden lehenrechtlichen Bestimmungen im Land- und Lehenrecht des Herzogtums Österreich siehe allgemein Luschin von Ebengreuth, Arnold: Handbuch der Österreichischen Reichsgeschichte, Band 1: Österreichische Reichsgeschichte des Mittelalters. Bamberg $1914{ }^{2}$, S. 147f.; Weltin, Max: Das österreichische Landrecht des 13. Jahrhunderts im Spiegel der Verfassungsentwicklung. In: Recht und Schrift im Mittelalter. Hg. von Peter Classen. Vorträge und Forschungen 23, 1977, S. 381-424, bes. 422f. Zur Diskussion um den „Leihezwang“ römisch-deutscher Kaiser und Könige siehe zusammenfassend Garnier, Claudia: Die Kultur der Bitte. Herrschaft und Kommunikation im mittelalterlichen Reich. Darmstadt 2008, S. 166-169. 
Lehensherrn ein Vorkaufsrecht zu, sollte sein Vasall sein Lehen zu verkaufen wünschen. Einem Verkauf an Dritte müsse der Herr nur zustimmen, wenn es sich bei dem Käufer um einen Verwandten (gesippten frewndt) handle. Möchte der Vasall sein Lehen verkaufen, weil er selbst erbenlos sei, so müsse der Herr diesem Kauf nur zustimmen, wenn der Verkäufer unter aid zu gott erkläre, zu dieser Veräußerung aufgrund einer persönlichen Notlage (leibsnarung halben) gezwungen zu sein. ${ }^{67}$

Über den rechtshistorischen Aspekt hinaus werfen diese Ausführungen die Frage auf, in welchen Fällen der habsburgische Landesfürst Schenkung, Verkauf oder Belastung von Lehengut duldete bzw. wann er sich gegen einen Lehenstransfer aussprach. Nähere Informationen über die Hintergründe von Lehensübertragungen an Dritte enthalten die in der habsburgischen Kanzlei des 15. Jahrhunderts in großer Zahl überlieferten Aufsandbriefe. ${ }^{68}$ Dabei handelt es sich grundsätzlich um Suppliken, d.h. Bittschriften, in denen der Petent seinem Lehensherrn die gewünschte Veräußerung mitteilt und um Belehnung des intendierten neuen Lehensinhabers ersucht. Diese Schreiben entsprechen in der Regel einem bestimmten Formular, dennoch finden sich zuweilen individuelle Ausführungen, etwa zu den Gründen, die den Verkauf eines Lehens notwendig gemacht hätten. So werden etwa die vom Vater des Lehensmannes verursachte hohe Schuldenlast $^{69}$ oder persönliche Schicksalsschläge als Ursachen für die finanzielle Notlage des Verkäufers herangezogen. ${ }^{70}$

Zuweilen erfolgen auch nähere Angaben über den gewünschten neuen Inhaber des Lehens, wenn etwa dessen große Frömmigkeit gepriesen oder dessen Status als langjähriger treuer Lehensmann betont wird ${ }^{71}$, wodurch man sich wohl eine günstigere Behandlung des Anliegens am kaiserlichen Hof erhoffte. Sicherlich zu demselben Zweck werden von den Petenten häufig landesfürstliche Vasallen oder einflussreiche Höflinge als Mitsiegler von Aufsandbriefen herangezogen oder um Unterstützung ihrer Anliegen am landesfürstlichen Hof ersucht ${ }^{72}$, während sich dem Kaiser nahestehende Personen bei einer intendierten Veräußerung ihres Lehensbesitzes mit einer knappen, formlosen Mitteilung an ihren Herrn begnügen konnten. ${ }^{73}$ Gelegentlich wird in Aufsandbriefen Ärger über die Weigerung des Herrn zur Zustimmung eines Verkaufs zum Ausdruck

67 HHStA Wien, Hs. B 7, f. 1r.

68 Zur Terminologie siehe etwa ebd., AUR 1481 XII 7, 1483 IV 25 (mein aufsand), 1493 V 31. Daneben erscheinen auch die zeitgenössischen Bezeichnungen aufgabbrief bzw. petbrief (ebd. 1474 VII 20, 1474 VII 25, 1491 V 26, 1491 VI 7).

69 Ebd. 1474 VII 25. Vgl. auch ebd. 1493 III 17 (Verkauf wegen mergklicher geltschuld).

70 Ebd. 1481 II 19 (Verkauf im Namen des Bruders, der auf der weit und nicht wissen, ob der in lebn oder tod ist), 1481 VII 14 (Verkauf von grosser [...] not wegen), 1476 XII 15, 1479 XII 4 und 1481 XII 7 (Verkauf aus merkhleiche $[r]$ notdurft), 1484 III 24 (Verkauf, um sunderlichen großen schaden abzuwenden).

71 Ebd. 1481 II 5 und 1481 XI 9.

72 Ebd. s.d. 1484 II 19; Fridericiana 5, Konv. 2, f. 80, und Fridericiana 6, Konv. 4, f. 82. Vgl. auch ein Schreiben Georgs von Frauenberg zu Haidenburg an den kaiserlichen Protonotar Waldner mit der Bitte um Förderung seines Anliegens; Tiroler Landesarchiv Innsbruck, Sigmundiana 14.572.

73 Siehe etwa ebd., AUR 1481 XII 7 (Aussteller: Bernhard von Tiernstein, landesfürstlicher Hauptmann im Viertel ob des Wienerwaldes). 
gebracht $^{74}$ oder vorhergehende erfolglose Suppliken in derartigen Angelegenheiten erwähnt. ${ }^{75}$ Neben der Einreichung von Bittschreiben blieb jedoch noch im späteren 15 . Jahrhundert weiterhin die persönliche, mündliche Aufsendung vor dem Lehensherrn möglich. ${ }^{76}$

Eine deutlich weitreichendere Verfügungsgewalt über Lehen besaß der Landesfürst beim Heimfall von Lehen, d.h. dem Rückfall in die Hand des Herrn. Dieser trat beim Tod eines Vasallen ohne lehensfähige Erben ein, darüber hinaus konnten Lehen auch nach freiwilliger Aufsagung durch den Vasallen oder strafweiser Einziehung anheimfallen - etwa in Folge von Treuelosigkeit gegenüber dem Lehensherrn, bei unentschuldigtem Versäumen des Lehensempfanges oder unrechtmäßiger Lehensveräußerung. ${ }^{77}$

Als Nutznießer der landesfürstlichen Lehenpolitik erscheint ein Kreis von Höflingen und Parteigängern, deren Dienste auch mit der Übertragung eingezogener Lehen abgegolten werden konnten. ${ }^{78}$ Darüber hinaus müssen zahlreiche landesfürstliche Urkunden, die Lehensübertragungen betreffen, im Zusammenhang mit der habsburgischen Kirchenpolitik gesehen werden, so etwa die häufig dokumentierte Umwandlung von landesfürstlichen Lehen in freies Eigen samt der darauffolgenden Stiftung durch die Inhaber an kirchliche Institutionen. ${ }^{79}$

Zusammenfassend muss trotz aller zeitlicher und regionaler Unterschiede, die bei einer umfassenderen Untersuchung des Lehenswesens in den habsburgischen Erblanden sicherlich stärker zu berücksichtigen wären, festgehalten werden, dass das vom Großteil der älteren Forschung vertretene Bild eines im Verfall befindlichen, von starrem Formalismus geprägten spätmittelalterlichen Lehenswesens, das für den Aufbau der Territorien keine Rolle gespielt habe ${ }^{80}$, auch auf dem Gebiet der habsburgischen Erblande einer umfangreichen Revision unterzogen werden muss. Mit dem Eindringen der Schriftlichkeit wird das Lehenswesen ein zentrales Betätigungsfeld der landesfürstlichen Kanzlei, dessen hoher Verwaltungsaufwand im 16. Jahrhundert zur Herausbildung eigener Lehenskanzleien für die habsburgischen Erbländer führte. Insbesondere bei

74 Ebd. 1472 XI 30: so mir ewr kayerliche gnad damit schuldig zetun ist.

75 Siehe etwa ebd. s.d. 1491 VIII 13 und 1492 VIII 17 (der Kaiser möge den genannten Besitz dem Käufer nu [...] genädigkleich geruechen zu verleichen).

76 Protokoll- und Urteilsbücher II, S. 716: so einer ein erkauffts lehen kauffen wolle, sull der, der das verkaufft, dem lehenheren mit mund und hanndt oder durch sein besigelt briefe aufgeben und den lehenheren bitten, das von im aufzunemen und dem kauffer zu verleihen [1472 April 13]. Siehe auch Regesten Kaiser Friedrichs III. Heft 14. Hg. von H. Koller, P.-J. Heinig und A. Niederstätter, bearb. von D. Rübsamen. Wien u.a. 2000, Nr. 370.

77 Siehe dazu Mitteis, H.: Lehnrecht, bes. S. 675-703; Diestelkamp, B.: Lehnrecht, S. 257f. ; Spieß, K.-H.: Lehnsrecht, S. 179; ders.: Lehnswesen, S. 54f. Zu Lehensentzügen durch habsburgische Landesfürsten im Spätmittelalter siehe etwa HHStA Wien, Hs. B 360, f. 216r-218v; Regesta Habsburgica V-2, Nr. 718; Regesten Kaiser Friedrichs III. Heft 12. Hg. von H. Koller und P.-J. Heinig, bearb. von T. Willich, Wien u.a. 1999, Nr. 317; ebd. Heft 30, Nr. 175.

78 Siehe etwa die Übertragung von dem Landesfürsten anheimgefallenen Lehen an die habsburgischen Kanzlisten Johann Waldner, Hans Halder und Georg Stadler: Tiroler Landesarchiv Innsbruck, Sigmundiana 14.547; HHStA Wien, AUR 1479 XI 28.

79 So etwa Regesta Habsburgica V-1, Nr. 191 und 232.

80 Vgl. zusammenfassend Schubert, Ernst: Fürstliche Herrschaft und Territorium im späten Mittelalter. Enzyklopädie deutscher Geschichte 35. München 2006², S. 71 (mit umfangreichen Literaturangaben). 
Herrschaftsteilungen und -wechseln wird deutlich, welch große Bedeutung der Vasallität noch im Spätmittelalter beigemessen wurde. Angesichts der zunehmenden Verdinglichung des Lehenswesens treten Vasallität und Territorialherrschaft nicht als Gegensätze auf, vielmehr erscheint die landesfürstliche Lehenshoheit im 14. und 15. Jahrhundert als ein nicht zu vernachlässigendes, wenn auch weitgehend monetarisiertes Mittel unter anderen zur Erweiterung und Konsolidierung landesfürstlicher Herrschaft.

\section{Zemský kníže jako lenní pán}

Výzkum lenního systému v pozdně středověkých habsburských dědičných zemích nepřesáhl doposud časově a teritoriálně omezené dílčí studie. Souvisí to především s představou staršího bádání o lenním systému v pozdním středověku jakožto úpadkovém a zcela zformalizovaném systému, který tehdy již nehrál při uplatňování reálné politické moci žádnou roli. Předložená studie nabízí především na základě diplomatických pramenů nová východiska pro posouzení zeměpanského lenního systému v Rakousích. Na příkladu lenních listin, lenních knih, reverzů a misiv je zřejmé, že se proces zlistinění stává důležitou náplní agendy panovnické kanceláře, jejímž dalším kvalitativním stupněm je vznik samostatné lenní kanceláře pro rakouské dědičné země v 16. století. Obzvláště v období dělené moci a při stř́ídání zeměpánů se ukazuje, jak velký význam byl vazalským vazbám přikládán. Vzhledem ke stále se rozrůstajícímu systému lenní soustavy nejsou rozhodně vazalské vztahy v rozporu s panovnickou mocí, naopak můžeme panovníkovu vrchní lenní pravomoc ve 14. a 15. století chápat jako jeden z monetárních prostředků k rozšiřování a konsolidaci této jeho moci. 
